\title{
DESIGN AND DEVELOPMENT OF A PLANAR INCHWORM ROBOT
}

\section{Song Huat Yeo I-Ming Chen R S Senanayake Peng Sin Wong}

\author{
School of Mechanical and Production Engineering \\ Nanyang Technological University \\ Nanyang Ave, Singapore 639798
}

\begin{abstract}
An inchworm-like robot is a mobile robot that imitates the locomotion pattern of a natural inchworm. Inchworm or snake like robots can crawl or move in highly constrained environment such as maintenance conduit for buildings and factories, and human intestines and blood vessels. In this paper, the development a planar inchworm robot based on the basic inchworm motion is presented. The motion is an extension of the basic crawling action of the inchworm but with further improvements to allow for greater flexibility and mobility for general planar motion. The unique mechanical arrangement of the actuators allows for quick change in travel direction and permits rotational movement. Pneumatic cylinders are used to achieve the motions instead of rotary motors. The robot is controlled through a personal computer with an interface card. It is showed that the proposed planar inchworm robot is able to move from a starting point to a destination within specified positional accuracy based on simple gait planning algorithm.
\end{abstract}

Keywords: Inchworm robot. Gait generation.

\section{INTRODUCTION}

Nature has provided researchers with an immerse variety of extremely skilled walkers and crawlers to study. Many investigations have been conducted on the motions of mammals and inserts whereby gait data are collected and analyzed. The fundamental objective of these locomotion studies is to transfer the knowledge gathered from gait analysis to the design of machines simulating these motions. A robotic prototype modelled directly from a mammal or insect will require complex and numerous joints and linkages. Modification and simplification to the motion concept are usually performed to minimise the actuators and joints needed. Ultimate aim would be to develop robotic movers with simple and minimal components that fulfil the movement criteria.

An inchworm-like robot is a mobile robot that imitates the locomotion pattern of a natural inchworm. The inchworm gait has been shown to be a feasible means of robot locomotion. Its movement exhibits desirable motion qualities like extreme stability, simplicity and the ability to travel in confined spaces. Successful inchworm robots have been developed in many research institutes. They can crawl or move in highly constrained environment such as maintenance conduit for buildings and factories, and human intestines and blood vessels $[1,2,3]$. Usually, these machines will move themselves in repetitive patterns, termed gaits, through the friction between the devices and the constrained environment.
With a good understanding of the basic inchworm motion, the immediate task would be to further develop motion concepts with additional flexibility in motion and speed of travelling. In this paper, the development a planar inchworm robot based on the basic inchworm motion is presented. The motion is an extension of the basic crawling action of the inchworm but with further improvements to allow for greater flexibility and mobility for general planar motion. The unique mechanical arrangement of the actuators allows for quick change in travel direction and permits rotational movement. All these are achieved without utilizing the traditional means of rotary motors. The robot draws its energy from pneumatic power and is controlled through a personal computer with an interface card.

\section{DESIGN OF INCHWORM ROBOTS}

\subsection{Basic inchworm}

Figure 1 shows the first prototype inchworm robot developed based on modular concept. Each module has a cart-like geometry moving along a horizontal track. There are two solenoid actuators on each of the cart: one for gripping action (emulating the gripper) and the other for extension action (emulating the extensor). The natural state of the gripper is retracted. It can be opened to hold on the wall. Viscous layers are adhered to the walls of the track to provide enough friction. The extensors are naturally retracted as well. Wheels are built under all carts to support the weight of the robot. The overall system is shown in Figure 2. This prototype is a simplified inchworm 
robot developed for gait analysis[4,5]. The robot is designed to travel along the guiding walls in both directions.

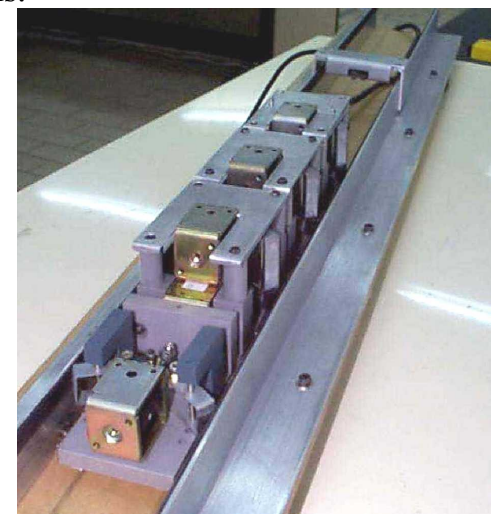

Figure 1: Inchworm Robot Platform

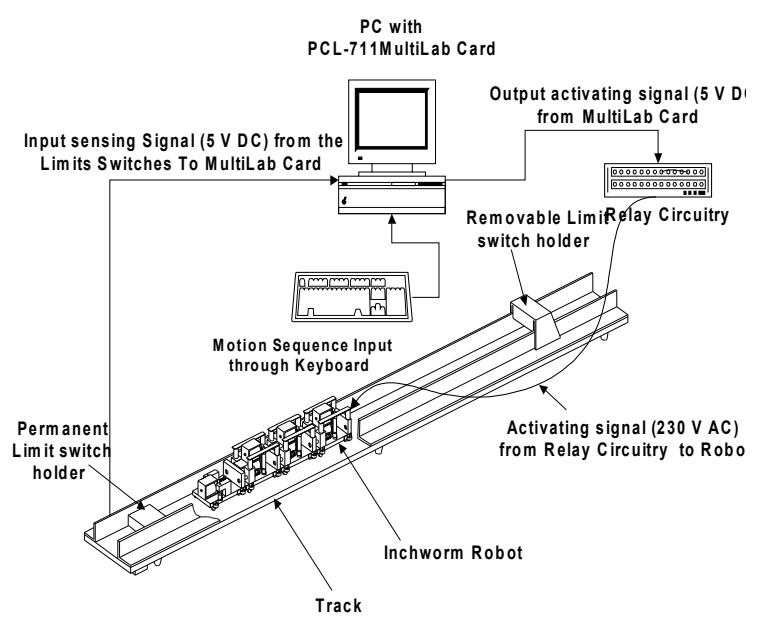

Figure 2: Inchworm robot system

With the above successful implementations of the basic inchworm motion, the advantage of the inchworm gait can be further exploited and modified to enable greater flexibility in "unbounded" planar motion.

\subsection{A two-dimensional planar inchworm robot}

To model a multi-directional planar robot based directly on the original inchworm body would require numerous flexible and elastic joints. Limitations foreseeable in this design include the difficulty in changing path direction, the lengthy time needed to execute the movements and the generation of turning motion. Turning movement, if possible would require a large turning radius.

A 2-dimensional model (planar) is introduced to extend the standard inchworm concept for general planar motion. The 2D planar model can allow for immediate change in travel direction and facilitate turning to a predefined angle. The concept retains the original inchworm gait of attaching at least one segment of the body to the travel surface before propelling the other segments forward.
Four actuators (Figure 3) are needed to perform this 2D-gait motion. These four actuators are linked together using pivot joints to form a square loop. At the four pivoting joints are grippers used to attach the pivot joints to the travel surface.

A combination of actuator extensions and retractions together with synchronised gripping action allow the execution of direct forward motion and rotational turning motion on a horizontal surface. Figure 3 illustrates the arrangement of the components.

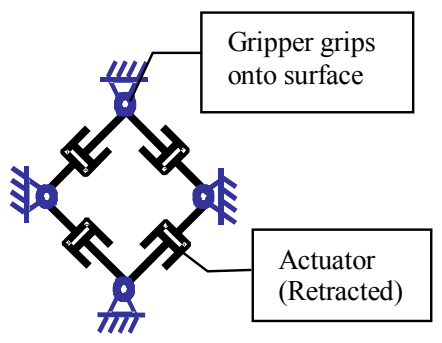

(a)

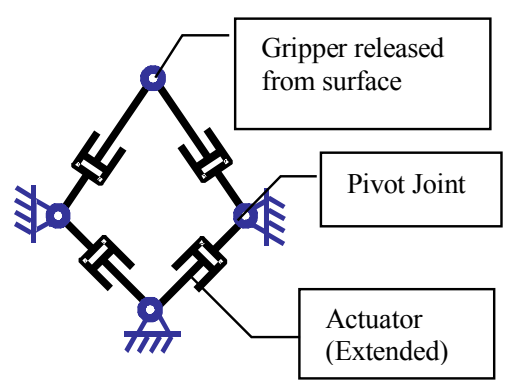

(b)

Figure 3: Components of Planar Inchworm Model

\subsection{Basic Gaits}

\subsubsection{Forward Translation Motion}

The complete sequence of a forward translation motion is illustrated in Figure 4. The robotic unit does not have a head or tail portion due to its square loop layout. Hence forward translation motion represents travel in the north, south, east and west directions relative to the robot body.

The sequence is for a step of forward translation motion based on a northward direction of travel. For forward translation in the other directions, similar sequence of actuation can be applied. The difference lies in the actuation of different sets of actuators.

\subsubsection{Turning Motion}

Figure 5 shows the execution sequences of actuation for a turning motion. The sequence is for a finite angle of rotation in the clockwise direction. For counterclockwise rotation, similar sequence of actuation can be applied but the corresponding sets of cylinders should be actuated. 


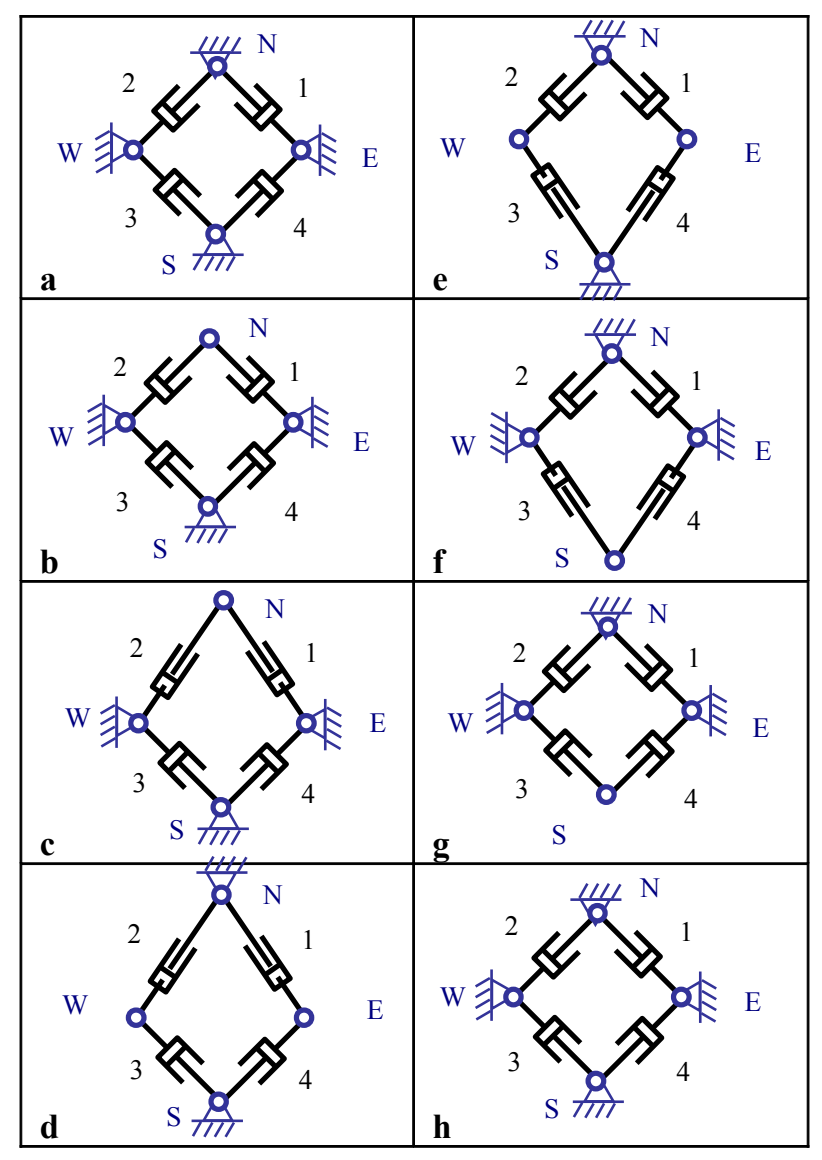

Figure 4: Forward translation motion

\subsection{Mechanical Design}

With the concept of the planar inchworm's motion sequence, the various mechanical designs to implement the required motion are generated. The basic functional requirements are:

- moves across a smooth flat surface at a reasonable speed;

- generates rotational motion in both clockwise and anti-clockwise direction;

- compact and the weight should not exceed $6 \mathrm{~kg}$;

- the size should be within a $50 \mathrm{~cm} \times 50 \mathrm{~cm}$ area;

- requires minimal setup time and maintenance after operation.

Figure 6 shows the outline of the mechanical arrangement of the proposed planar inchworm robot. Four pneumatic cylinders are located on a cross bar frame in a square format. The ends of the cylinders are jointed to four slide units which can move with respect to the cross bar frame in which they are positioned. Suction pads are used to enable the planar inchworm robot to secure itself to the surface and wheels are added for mobility.

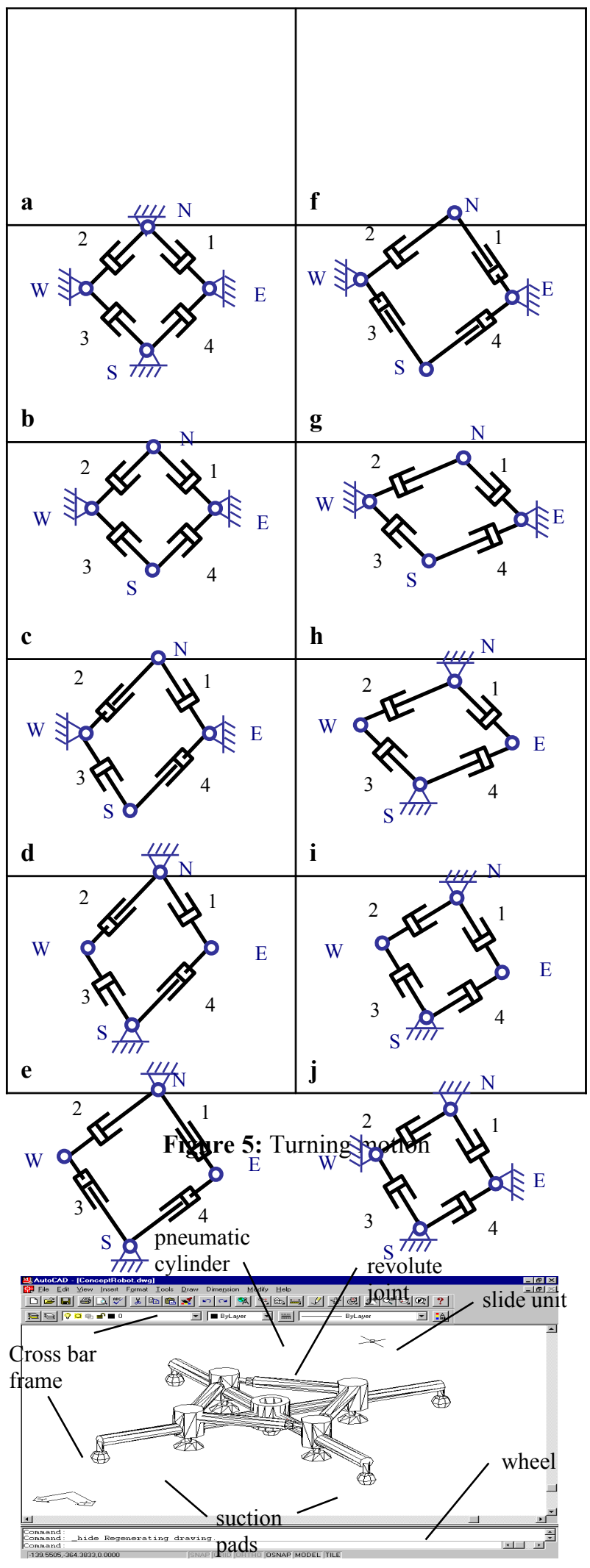

Figure 6: Mechanical arrangement of cylinders

The planar inchworm robot as shown in Figure 7 is built and tested in the laboratory [6]. The 4 pneumatic actuators on the cross bar arrangement and suction pads can be clearly seen in Figure 8 . 


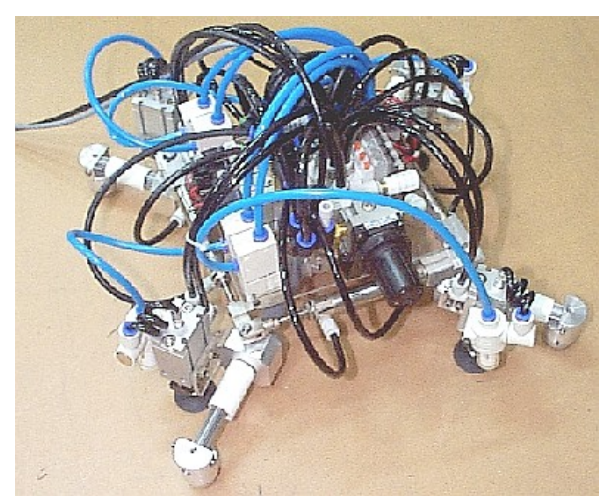

Figure 7: Fabricated planar inchworm robot

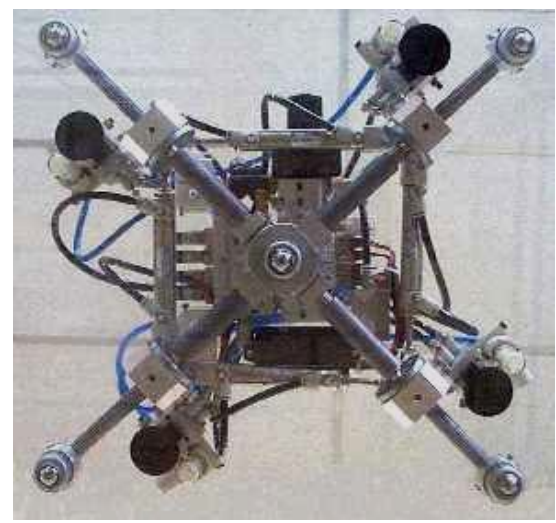

Figure 8: Underside view of planar inchworm robot

The following observations are noted from tests carried out on the planar inchworm robot:

- The planar inchworm robot takes about 25 seconds to execute the forward motion sequence and 30 seconds for the rotational motion.

- When placed on an inclined plane made of perspex, the planar inchworm robot is able to travel successfully in all directions and execute turning movements at an incline angle of up to $30^{\circ}$. At greater incline angle, it starts slipping during certain transformation. The ability to travel on inclined surface demonstrates the viability of a wall-climbing model based on the present prototype.

- A slightly jerky motion is encountered initially when the rates of extension and retraction between the four motion cylinders are different though the end positioning is not affected. However, with proper tuning and adjustment, the transitional motion was brought to a smoother level.

- At the "home' arrangement, the shape of the four motion pistons should be in a square formation. However it is found that after a number of motion transformations, the square arrangement is slightly "distorted". To prevent this from happening, shape restriction pipes (shown in Figure 9) are added to the cross bar frame to limit the pistons arrangements ensure a proper "home" arrangement is reached at the end of

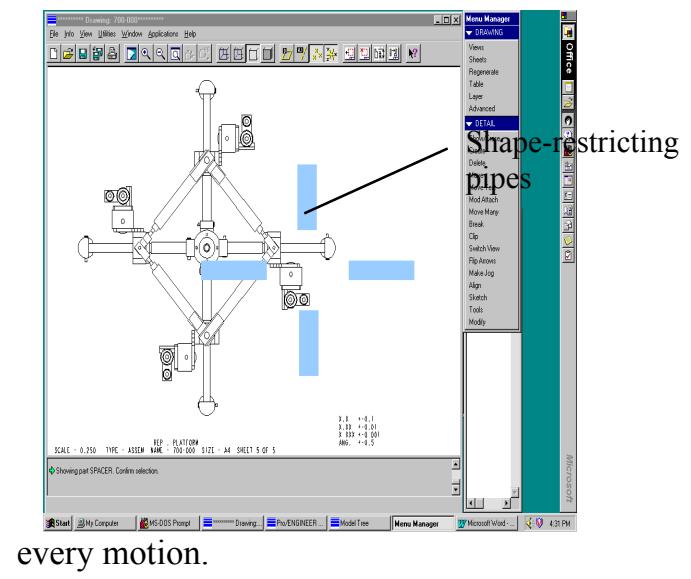

Figure 9: Shape-restricting pipes

\section{SIMPLE GAIT PLANNING}

A gait planning algorithm is required in order to move the planar inchworm robot from a starting position to a destination point $D$. Clearly, it can arrive at Point $D$ accurately or at least within a small tolerance. Theoretically, there are an infinite number of paths that can satisfy this criterion. Here, it is assumed that the planar inchworm robot does not travel beyond the quadrant of the coordinate system in which point $D$ lies, and that the inchworm moves along one of its four main axes. Rotation angles are then measured with respect to that travelling axis. A simple gait planning that minimizes the positional error is implemented [7] that allows the planar inchworm robot to move from a starting position to a destination point $D$ within a specified tolerance.

Figure 10 shows the iteration results. using the search algorithm in finding a suitable path. In this example, point $D$ is at $X=300 \mathrm{~mm}$ and $Y=200 \mathrm{~mm}$. The stride length $L$ of the planar inchworm robot is $60 \mathrm{~mm}$ and the rotation angle is $12.5^{\circ}$. A feasible solution would be one that yields an error of less than $1 \mathrm{~mm}$.

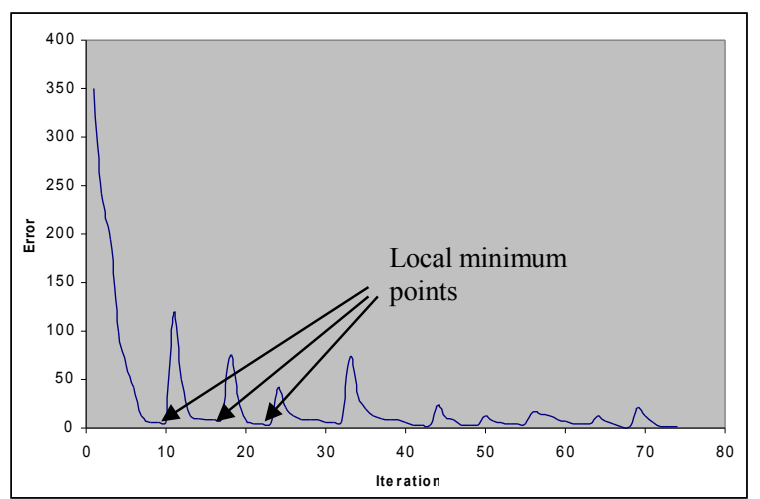


Figure 10: Iteration results

It is seen that at the beginning of the search routine, the error is very large. The error decreases almost exponentially in the next 10 iterations. Then the function reaches a local minimum point. The program then resets some of the initial values and search continues. The surge in the error is due to this resetting but the new errors are not as high as before.

The search routine finds several local minimum points before choosing a feasible solution. The total number of iterations in this case is 75 , and the final positional error is $0.89 \mathrm{~mm}$.

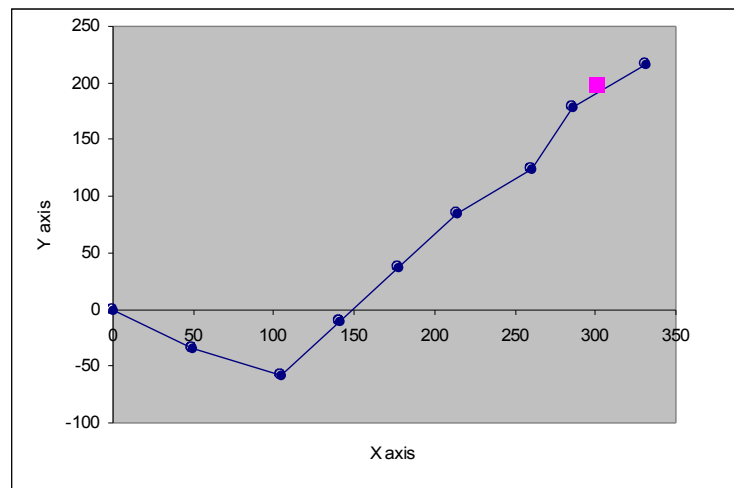

Figure 11: Path planned with $8^{\text {th }}$ iteration

Figure 11 shows the path computed at $8^{\text {th }}$ iteration. The destination point $D$ is marked by a square. The error using the path is about $30 \mathrm{~mm}$. Figure 12 shows the comuted path after $42^{\text {nd }}$ iteration and the error is reduced to $10 \mathrm{~mm}$. Figure 13 shows the path taken by the planar robot using the 72th iteration with an error of $0.89 \mathrm{~mm}$.

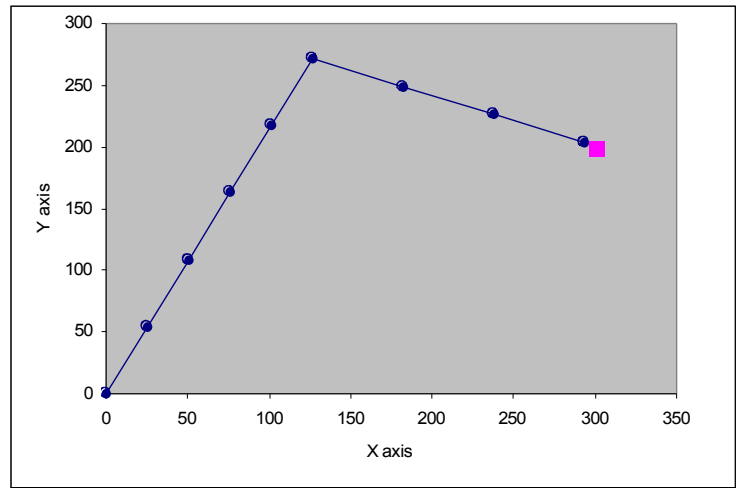

Figure 12: Path planned with 42 iterations

In this example, the region estimation method is not used since Point $D$ is not very far away and the number of design variables is not large. Figure 14 shows a series of snap shots of the planr robot in motion according to the computed path.

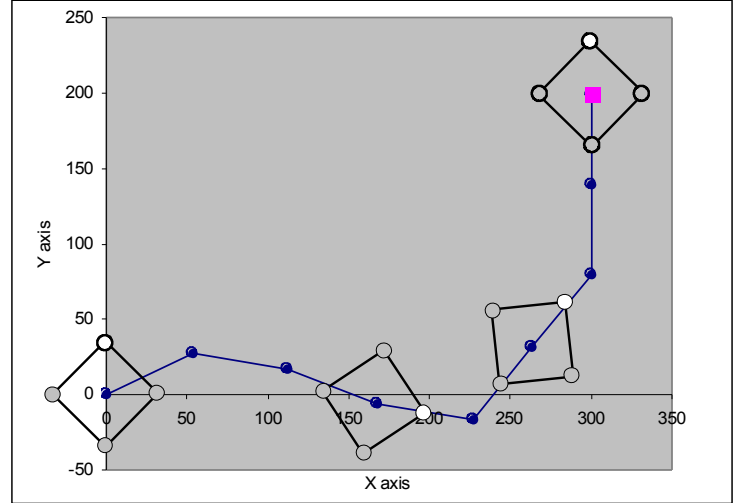

Figure 13: Final path taken

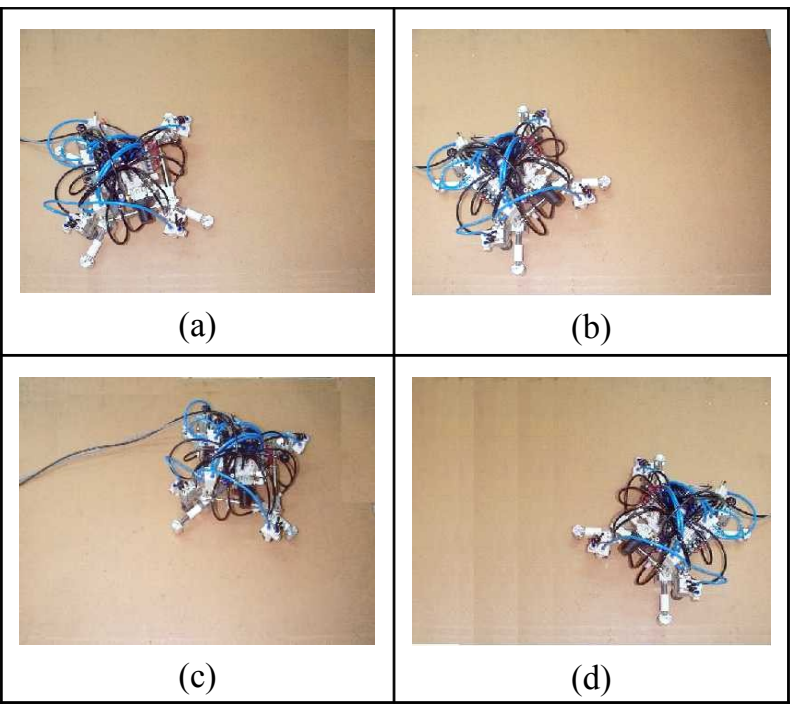

Figure 14: Planar inchworm robot in motion

\section{CONCLUSION}

In this paper, the extension of the standard inchworm concept for general planar motion is presented and the development of a planar inchworm is described. The concept of movement for the robot is derived from the generic crawling motion of the inchworm but is farther improvised to attain greater flexibility and mobility. This inchworm planar concept of movement provides a useful alternative to the traditional means of motion generation methods. The 2D planar model can both translate in step of $60 \mathrm{~mm}$ along one of its main axis and rotate at a finite angle of $12.5^{0}$.

This prototype robot can achieve stable motion with the ability to execute quick direction change and rotation movements. Furthermore, the planar inchworm robot can also travel on an inclined surface, indicating the viability of using it for wall climbing. The successful working prototype brings to reality a conceptual motion idea that was previously limited to simulations and modelling. 
The chosen hardware was based on operating requirements to suit the research laboratory environment. Alternative forms of suitable actuators or grippers can also be used to generate the planar inchworm motion concept.

Further development based on the current prototype includes:

(a) Wall climbing model

Wall climbing capabilities can be achieved by modifying the current model. To increase the suction grip force for vertical climbing, a larger diameter suction pad is recommended. To lesser the weight of the unit, the vacuum generation components and solenoid valves can be dismounted from the body. The climbing model will consist of only the basic structure and motion pistons. A vacuum supply tube will bring suction power from the ground.

(b) Autonomous model

An autonomous version can be constructed using electrical actuators, on board micro-controllers, and dry cells. The application of electrical motion actuators eliminates the need for compressed air supply. The gripping action can be attained from electro-magnets. Remote control operation completes the free ranging autonomous model.

(c) Intelligent model

A variety of sensors can be installed onto the robot to provide intelligence capability. Obstacle avoidance and path recognition are possible functions that can be obtained with ultrasonic sensors, machine visions and the proper software. A video camera may be incorporated for monitoring the robot orientation and processes.

\section{ACKNOWLEDGEMENT}

The authors would like to thank Mr Edwin Ho, Miss Gao Yan \& Mr Ting Teck Beng for their help in the inchworm project.

\section{REFERENCES}

[1] Carrozza M.C., Lencioni L., B. Magnani, P. Dario, D. Reynaerts, M.G. Trivella, A. Pietrabissa, $A$ Microrobot for Colonoscopy, $7^{\text {th }}$ Int. Sym. On Micro Machine and Human Science, 1996.

[2] Slatkin A.B., Burdick J.W., The Development of a Robotic Endoscope, Proceedings IEEE/RSJ Int. Conf. Intelligent Robots Systems, pp162-171, 1995.

[3] Fukuda T., Hosokal H., Uemura M., Rubber Gas Actuator Driven by Hydrogen Storage Alloy for InPipe Inspection Mobile Robot with Flexible Structure, Proceedings IEEE Conf. Robotics Automation, pp1847-1852, 1989.

[4] Gao Y., Gait Generation and Planning for Inchworm-like Robot Locomotion, Technical Report, School of Mechanical \& Production Engineering, Nanyang Technological University, 1998.

[5] I.M. Chen, S.H. Yeo, H.L. Ho, Y. Gao, Gait Generation for Multi-Segment Inchworm-Like Robot Locomotion, Video proceedings IEEE Int. Conf. Robotics Automation, Detroit, MI, USA, 1999.

[6] Wong P.S., Design and Fabrication of A Planner Inchworm Robot, FYP Report, School of Mechanical and Production Engineering, Nanyang Technological University, 1999.

[7] Ting T.B., Locomotion Gait Planning for a Planar Inchworm Robot, FYP Report, School of Mechanical and Production Engineering, Nanyang Technological University, 2000. 